\section{Polyacrylamide Gel Electrophoresis (PAGE) of Synthetic Nucleic Acids}

This unit describes methods and protocols for denaturing polyacrylamide gel electrophoresis (PAGE) for the analysis and purification of synthetic oligonucleotides. The analytical information is nonquantitative and recorded usually by a photograph (UV shadow or staining), autoradiography by radiolabeling ( СPMB UNITS $3.10 \& 6.4$ ), or digital methods, e.g., densitometry scanning.

The materials and equipment for PAGE are relatively inexpensive and available in most molecular biology laboratories. The most convenient and familiar format, the vertical slab gel apparatus, is commercially available in different dimensions and configurations. The cross-linked polyacrylamide gel matrix is held between two glass plates and exposed on the top and bottom to chambers containing buffered electrolyte solution through which an electric field of controlled voltage or current is applied.

The major operations involved in conducting PAGE are (1) sample preparation, (2) electrophoresis, (3) visualization of the gel, and (4) isolation of the product (purification). The protocols for analysis (see Basic Protocol 1) differ from those used for purification (see Basic Protocol 2); therefore, they are discussed separately. One method of staining gels for purposes of visualization is described in Support Protocol 1; radiolabeling of oligonucleotides (prior to separation by PAGE) for visualization by autoradiography is described in Support Protocol 2.

Oligonucleotides made by the automated phosphoramidite method (UNIT $3.3 \&$ APPENDIX $3 C$ ) should be quantitated prior to PAGE by UV absorbance $\left(A_{260}\right)$ so as not to overload or underload the gel. Initial analysis by MALDI-TOF (UNIT 10.1) or electrospray mass spectrometry analysis (UNIT 10.2) may be advised in some laboratories to ensure the presence of the full-length oligonucleotide.

CAUTION: Radioactive materials require special training and handling; all supernatants must be considered radioactive waste and disposed of appropriately. $\left[\gamma_{-}{ }^{32} \mathrm{P}\right]$ ATP should be handled behind a $\beta$ shield.

CAUTION: Acrylamide and bisacrylamide monomers are hazardous. Solutions of acrylamide deteriorate quickly, especially when exposed to light or left at room temperature.

\section{ANALYSIS OF SYNTHETIC NUCLEIC ACIDS BY PAGE}

The steps in PAGE analysis of oligonucleotides include (1) preparation of the gel and setup of the gel apparatus, (2) electrophoretic separation, and (3) detection of the oligonucleotides on the gel. There are two alternatives for detection--staining with methylene blue or SYBR Green, and radiolabeling analysis. For radiolabeling analysis, the oligonucleotides are first labeled at the $5^{\prime}$ end using $\left[\gamma^{32} \mathrm{P}\right]$ ATP and T4 polynucleotide kinase. Following electrophoresis, the radiolabeled oligonucleotides are detected by exposing the gel to X-ray film (autoradiography) or by use of a PhosphorImager (Molecular Dynamics; not described here).

\section{Materials}

$38 \%(\mathrm{w} / \mathrm{v})$ acrylamide/2\% (w/v) bisacrylamide (see recipe)

$1 \times$ formamide/dye mix (see recipe for $10 \times$; dilute in TBE buffer)

Loading buffer: 9:1 formamide/1× TBE buffer
BASIC

PROTOCOL 1

Purification and Analysis of Synthetic Nucleic Acids and Components 
Vertical electrophoresis system, such as Hoefer Model SE 400 or SE 600 in the 16

$\times 18-\mathrm{cm}$ format with power supply (Pharmacia Biotech)

Additional reagents and equipment for denaturing polyacrylamide gel electrophoresis (APPENDIX $3 B)$

\section{Set up gel}

1. Assemble the gel plates, spacers, and combs as described in APPENDIX $3 B$, or following manufacturer's instructions.

2. Determine the optimum acrylamide concentration for the range of oligonucleotide lengths as shown in Table 10.4.1. Prepare the desired acrylamide gel solution as described in APPENDIX $3 B$ in a side-arm Erlenmeyer flask, adding everything but the TEMED.

3. Stopper the flask and apply a weak vacuum (using a water aspirator) through the side arm for $\sim 5$ min to degas and remove oxygen.

4. Vent the system and add $50 \mu \mathrm{L}$ TEMED while mixing. After $30 \mathrm{sec}$, pour the gel(s) as described in APPENDIX $3 B$.

A 10- to 25-mL pipet with a rubber bulb is a convenient dispensing device.

5. Insert the comb. Dislodge any trapped air bubbles, especially in the wells, by tapping gently on the glass plates.

\section{Perform electrophoresis}

6. After gel has polymerized (usually for $\geq 1 \mathrm{hr}$ ), remove bottom spacer of gel sandwich. Remove extraneous polyacrylamide from around combs. Clean spilled urea and acrylamide solution from outer plate surfaces with water.

If gels are to be stored for several days before use, remove the comb upon polymerization, fill the wells with water, and place a piece of Parafilm or UV-transparent plastic wrap over the top of the gel sandwich to prevent drying.

For further detail regarding gel preparation and handling see APPENDIX $3 B$.

7. Place the gel on the electrophoresis apparatus. Add enough $1 \times \mathrm{TBE}$ buffer to the upper and lower chambers, to submerge the gel and wells by $\sim 3 \mathrm{~cm}$.

8. Flush the wells with $1 \times$ TBE buffer using a Pasteur pipet to remove debris and urea before the sample is applied.

9. Set the power supply to a constant power of 20 to $40 \mathrm{~W}$ (for a $16 \times 18-\mathrm{cm}$ format).

For other formats, set the power to draw 20 to $40 \mathrm{~V} / \mathrm{cm}$.The ideal wattage of the gel should generate enough heat so that the gel plates are warm, but not too hot to touch $\left(\sim 50^{\circ} \mathrm{C}\right)$.

10. Prerun with a blank sample of 5 to $10 \mu \mathrm{L}$ of $1 \times$ formamide/dye mix in any one or more of the lanes for $\sim 30 \mathrm{~min}$ to ensure the lanes are running straight and to predict the time required for appropriate sample migration.

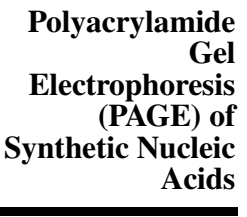

10.4 .2

Table 10.4.1 Selecting the Acrylamide Concentration

\begin{tabular}{cc}
\hline Oligonucleotide size $(\mathrm{nt})$ & Polyacrylamide concentration $(\%)$ \\
\hline$<25$ & 20 \\
$25-50$ & 15 \\
$50-90$ & 12 \\
$>90$ & 8 \\
\hline
\end{tabular}


Prerunning also increases the temperature of the gel, which helps to denature secondary structures, elutes impurities from the gel, and serves as visible confirmation that the gel is functioning properly.

11. Dissolve each sample oligonucleotide ( 0.5 to $2 \mathrm{OD}$ units) in $10 \mu \mathrm{L}$ of loading buffer by vortexing. Microcentrifuge the dissolved sample briefly to collect it at the bottom of the microcentrifuge tube.

These conditions are appropriate where visualization is to be done by UV shadowing or staining analysis (using a 0.8-mm thick gel). For detection by autoradiography, load 2 to 20 pmol of crude radiolabeled oligonucleotide (5 to $10 \mu \mathrm{L}$ of labeling reaction; Support Protocol 2) on a 0.4-mm thick, 40-cm long gel.

12. Flush the sample wells again with $1 \times$ TBE buffer and load the samples under the surface of the buffer and just above the surface of the well.

A small, 10- to 25- $\mu L$ syringe with a 25-G needle, or a flat-tipped micropipettor can be used to load the sample.

13. Start electrophoresis immediately using the conditions described in step 9.

Electrophoresis should be started immediately after loading, to avoid band broadening by diffusion

14. Run until the position of the marker dye indicates that the DNA has been sufficiently resolved. For radiolabeled samples, dry the gel and perform autoradiography as described in APPENDIX $3 B$. To visualize unlabeled oligonucleotides by methylene blue staining, see Support Protocol 1. For detection by UV shadowing, see Basic Protocol 2 , steps 5 to 11 .

Oligonucleotides longer than $\sim 80$ nt often do not form well-shaped, detectable product bands, and should be analyzed by radiolabeling/PAGE or gel capillary electrophoresis. A photograph can be taken under UV light for a permanent record.

\section{VISUALIZATION USING METHYLENE BLUE STAINING}

Direct visualization in the gel matrix can be done with a DNA-specific stain such as methylene blue or SYBR Green (Molecular Probes, 1996). Staining can be particularly useful if the gel is to be preserved by drying, but is not recommended for preparative purposes when the analyzed sample is to be used in subsequent applications. As an analytical tool, staining has limitations because some sequences in low concentrations may not be visible. Nevertheless, staining is more sensitive than UV shadowing and is considerably easier than radiolabeling. The stained gel can be photographed under ambient light, as opposed to short-wavelength UV light.

\section{Additional Materials (also see Basic Protocol 1)}

$0.02 \%$ (w/v) methylene blue (Aldrich) in $\mathrm{H}_{2} \mathrm{O}$

1. Electrophorese $\sim 1$ OD unit of oligonucleotide per lane until the product has migrated about two-thirds down the gel (use bromphenol blue and xylene cyanol FF as marker dyes).

2. Disassemble the gel sandwich and carefully place the gel in a shallow pan containing enough $0.02 \%$ methylene blue to cover the gel.

3. Let stand for 15 to $30 \mathrm{~min}$, then decant the stain and gently rinse the gel in water.

If the background staining is too high, some destaining can be effected by soaking the gel in water.

4. Photograph the gel under ambient light against a white background.

Purification and Analysis of Synthetic Nucleic Acids and Components 
SUPPORT PROTOCOL 2

\section{RADIOLABELING OF OLIGONUCLEOTIDES USING T4 POLYNUCLEOTIDE KINASE}

It is not necessary to prepurify or desalt sample oligonucleotides for radiolabeling analysis, as they may be used after drying down directly from the ammonia deprotection solution. The amount of each sample to be labeled can vary according to the activity of the $\left[{ }^{32} \mathrm{P}\right]$ ATP and the desired autoradiography film development time. The following protocol provides for loading samples in duplicate and will result in an approximate film development time of $\sim 1$ to $2 \mathrm{hr}$.

Label the following quantities of oligonucleotides according to length for $5^{\prime}{ }^{32} \mathrm{P}$-radiolabeling analysis:

$<40$ nt: 5 pmol;

40 to 70 nt: 10 pmol;

$>70$ nt: 20 to 50 pmol.

Convert absorbance to molar quantities by the approximation of $1 \mu \mathrm{mol}$ oligonucleotide $=10 \mathrm{OD}$ units/nt. For example, $5 \mathrm{pmol}$ of a 20 -mer is $0.001 \mathrm{OD}$ unit. Typically 2 to 20 pmol of crude radiolabeled oligonucleotide are loaded onto a $0.4-\mathrm{mm}$ thick, $40-\mathrm{cm}$ long gel for optimum resolution. It will be necessary to make a serial dilution of the sample in water to avoid pipetting submicroliter aliquots.

\section{Materials}

Radiolabeling master mix (see recipe)

$10 \times$ formamide/dye mix (see recipe)

Additional reagents and equipment for preparing and running a polyacrylamide gel (Basic Protocol 1)

1. Dry quantity of oligonucleotide to be labeled in a microcentrifuge tube.

2. Vortex the master mix and microcentifuge briefly to collect any drops from the sides of the tube.

3. Pipet $6 \mu \mathrm{L}$ of the master mix into each sample tube.

4. Vortex, then microcentrifuge briefly. Incubate at $37^{\circ} \mathrm{C}$ for 45 to $60 \mathrm{~min}$.

5. Chill on ice for $\sim 2 \mathrm{~min}$, then microcentrifuge briefly. Add $14 \mu \mathrm{L}$ of $10 \times$ formamide/dye and mix. Vortex, then microcentrifuge briefly.

6. Load 5 to $10 \mu \mathrm{L}$ of each sample into the flushed wells of a prerun gel (see Basic Protocol 1).

The dye-containing samples are stable, may be stored at $4^{\circ} \mathrm{C} /-20^{\circ} \mathrm{C}$ for weeks, and are ready for loading on the gel. With a half-life of 14 days, ${ }^{32} P$-labeled oligonucleotides should be used within a few weeks.

BASIC PROTOCOL 2

Polyacrylamide

Electrophoresis (PAGE) of

Synthetic Nucleic Acids

\section{PURIFICATION OF SYNTHETIC NUCLEIC ACIDS USING PAGE AND UV SHADOWING}

The key steps in the PAGE purification process are (1) electrophoretic separation, (2) UV-shadow visualization, (3) excision of the product band from the gel, and (4) recovery of the pure product from the gel matrix (Efcavitch, 1990; Andrus, 1992; Ellington and Pollard, 1998). Crude samples of long oligonucleotides (>50 nt) contain substantially more truncated sequences due to the increased number of coupling cycles. A crude long 
oligonucleotide is in a lower-purity state than a shorter one, therefore, with a long oligonucleotide, more sample must be loaded on a preparative gel to visualize and isolate a useful amount. There is a limit to how much oligonucleotide can be loaded on a gel before resolution is lost and distortion prevails. Desalting prior to purification is recommended, e.g., by precipitation, for oligonucleotides $>50 \mathrm{nt}$.

\section{Materials (also see Basic Protocol 1 and APPENDIX $3 в$ )}

$20 \times 20-\mathrm{cm}$ fluorescent TLC plate, wrapped with UV-transparent plastic wrap Hand-held, short-wavelength UV light (240 to $300 \mathrm{~nm}$ )

1. Prepare a denaturing polyacrylamide gel as described in Basic Protocol 1 and APPENDIX $3 B$.

The highest percentage gel for a given length is recommended to obtain maximum resolution (Table 10.4.1). Typically, 1.5- to 3.0-mm thick preparative gels are used. Longer oligonucleotides require longer migration distances, i.e., longer gels, to obtain sufficient resolution. The 16-cm height gel for oligonucleotides up to $50 \mathrm{nt}$ and 40-cm gel height for oligonucleotides $>50 \mathrm{nt}$ are useful. The width of the comb teeth should be 2 to $3 \mathrm{~cm}$ and the maximum sample loading is $\sim 10$ to 20 OD units per lane for the aforementioned dimensions.

2. Quantitate and prepare the DNA samples for purification as for analysis (see Basic Protocol 1). Dissolve the dried oligonucleotide sample in loading buffer to a concentration of $\sim 1$ to 2 OD units $/ \mu \mathrm{L}$.

Prepurification desalting is recommended to maximize resolution, e.g., precipitation, for $>50$ nt. Overloading a gel can lead to considerable distortion. Samples that do not readily dissolve in the loading buffer should be heated to $\sim 60^{\circ} \mathrm{C}$ and mixed. It is recommended that tracking dyes not be loaded in the same lane as the sample to avoid possible contamination and masking of the sample during UV shadowing.

3. Load samples and tracking dyes on the gel. Load tracking dyes in the outermost wells of the gel as a gauge to monitor the migration of the oligonucleotide.

The crude long oligonucleotide is in a lower-purity state than a shorter one; it is necessary to load a larger sample of a long oligonucleotide on a preparative gel so that the product can be visualized and isolated in a useful amount.

4. Run the gel as described in Basic Protocol 1, steps 9 to 13.

The gel conditions and concentrations are essentially the same for purification as they are for analysis, although the thicker gels require a higher power setting. The product oligonucleotide to be isolated should migrate as far down the gel as possible to achieve optimum product separation. This is unlike analysis, where more of the faster-running failure sequences need to be observed. Because the purpose is to separate the oligonucleotide from the crude mixture, it is acceptable to run the failure sequences off the bottom of the gel. Ideally the product band should be allowed to migrate two-thirds or more of the length of the gel. As a guideline, refer to the migration of dyes and oligonucleotides versus acrylamide concentration shown in Figure 10.4.1.

5. Remove the gel from the glass plates and place on top of a fluorescent TLC plate covered with UV-transparent plastic wrap.

6. Visualize the oligonucleotide under a short-wavelength UV light by holding the lamp directly overhead. The UV-absorbing oligonucleotide masks the fluorescent emission of the plate and appears as a shadow.

Minimize the exposure of the oligonucleotide to UV light, which can cause degradation.

7. Slice the gel on the perimeter of the product band with a clean razor blade.

Purification and Analysis of Synthetic Nucleic Acids and Components 


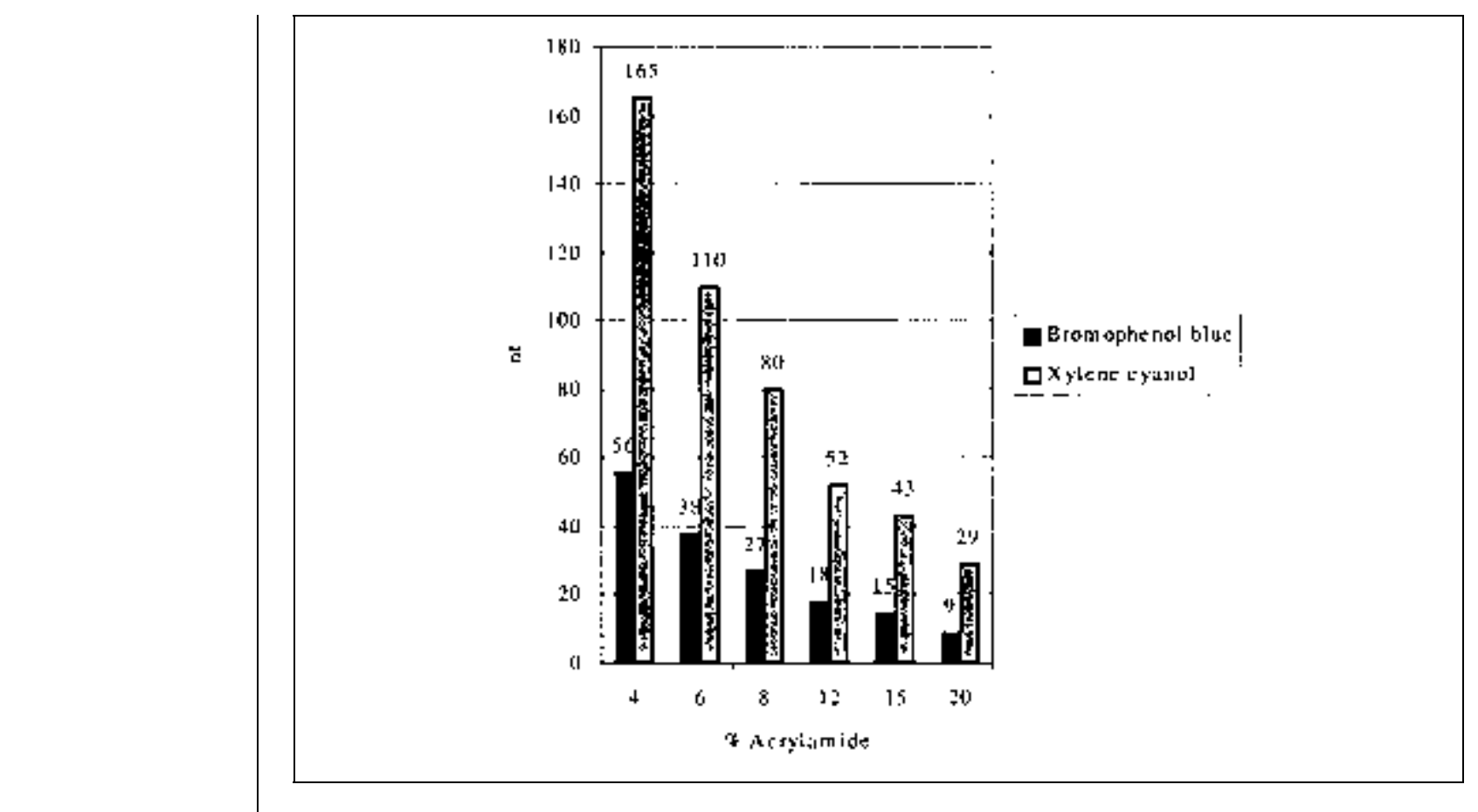

Figure 10.4.1 Migration of dyes and oligonucleotides versus acrylamide concentration.

If the oligonucleotide does not have mixed-base sites, the cuts should be slightly to the interior of the product band to eliminate $N$ - 1 contamination. To avoid missing some of the possible sequences in a degenerate product mixture, the cuts should be be at or slightly outside of the diffuse product band.

8. Transfer the excised gel fragment(s) with tweezers to a small vessel (e.g., 15-mL conical, disposable tube) and crush the fragments with a spatula or rod.

If more than one sample is run on the same gel, use caution when handling, to prevent cross-contamination.

9. Add just enough extraction solution (water or an aqueous, neutral buffer, such as 50 $\mathrm{mM}$ triethylammonium acetate) to cover the gel fragment. Vortex briefly and let stand for 8 to $24 \mathrm{hr}$ at room temperature. Alternatively, the oligonucleotide can be isolated from the gel fragment by electroelution (UNIT 5.4).

10. Withdraw the oligonucleotide-containing solution and desalt by precipitation, sizeexclusion filtration, or affinity cartridge protocols (UNIT 10.6).

11. Quantitate by UV absorbance. Typical recovery yields vary somewhat, but are usually $\sim 50 \%$.

Polyacrylamide (PAGE) of Synthetic Nucleic Acids 
REAGENTS AND SOLUTIONS

Use distilled, deionized water or other ultrapure water in all recipes and protocol steps. For common stock solutions, see APPENDIX 2A; for suppliers, see SUPPLIERS APPENDIX.

Acrylamide/bisacrylamide, $38 \%(w / v) / 2 \%(w / v)$

Dissolve $380 \mathrm{~g}$ acrylamide and $20 \mathrm{~g}$ bisacrylamide in deionized water to a volume of $1 \mathrm{~L}$. Filter through a $0.5 \mu \mathrm{M}$ membrane and store at $4^{\circ} \mathrm{C}$, protected from light.

Formamideldye mix, 10 $\times$

Prepare in deionized formamide:

$0.1 \%(\mathrm{w} / \mathrm{v})$ bromphenol blue

$0.1 \%(\mathrm{w} / \mathrm{v})$ xylene cyanol FF

Store up to 3 months at $-20^{\circ} \mathrm{C}$

Kinase buffer, 10×

$0.25 \mathrm{M}$ Tris.Cl, pH 7.6 (APPENDIX 2A)

$0.1 \mathrm{M} \mathrm{MgCl}_{2}$

$0.1 \mathrm{M} \mathrm{DTT}$

Store at $-20^{\circ} \mathrm{C}$; discard after 6 months

\section{Radiolabeling master mix}

For each sample, prepare a master mix consisting of the following:

$4 \mu \mathrm{L}$ of $100 \mu \mathrm{M}$ cold ATP in water $(400 \mathrm{pmol})$

$1 \mu \mathrm{L}$ of kinase buffer (see recipe)

$0.2 \mu \mathrm{L} 10 \mathrm{mM}$ spermidine

$1 \mu \mathrm{L}(10 \mathrm{U}) \mathrm{T} 4$ polynucleotide kinase (New England Biolabs)

$1.5 \mu \mathrm{L} 5 \mathrm{mCi} / \mathrm{ml}\left[\gamma^{-}{ }^{32} \mathrm{P}\right] \mathrm{ATP}$ [(3000 Ci; $\left.111 \mathrm{TBq}\right] / \mathrm{mmol}$; NEN Life Science Products) $]$

\section{COMMENTARY}

\section{Background Information}

Charged molecules, such as oligonucleotides, migrate through the gel matrix under the influence of the applied electric field. The exact equation for electrophoretic velocity is complex, but the overwhelming factors are charge and mass. The charge-to-mass ratio for oligonucleotides remains virtually constant, independently of length. Molecular shape, net hydrophobicity, size, interactions with the gel matrix, and other parameters allow separation due to the net difference in velocity of sieving through the gel matrix. The elution order of an inverse relationship between length and velocity is established at $\sim 10 \mathrm{nt}$ in length. For very short oligonucleotides, from 2 to $\sim 10 \mathrm{nt}$, the charge-to-mass ratio changes rapidly and the elution order is reversed. For example, a dimer migrates very slowly and a trimer slightly faster under denaturing gel conditions $(7 \mathrm{M}$ urea). Synthetic oligonucleotides of common lengths between 15 and $50 \mathrm{nt}$ generally separate into regular, predictable band patterns in a lane on the polyacrylamide slab gel.

The length, and, to a lesser degree, the sequence of an oligonucleotide affect the mobil- ity of DNA through the gel. The order of migration contributed by the bases is $\mathrm{C}>\mathrm{A}>\mathrm{T}>\mathrm{G}$, with $\mathrm{C}$ being the fastest (Maniatis et al., 1975; Efcavitch, 1990). Oligonucleotides of the same length but with different sequence and base content will not migrate at exactly the same rate. Therefore, comparison of an "oligonucleotide size marker" with the sample oligonucleotide to verify the correct length gives only an approximate comparison.

Denaturing conditions should be maintained with single-stranded DNA (oligonucleotides) to minimize the formation of duplexes and other secondary structures. Secondary structures can significantly alter the expected mobility of an oligonucleotide. Both the high concentration of urea $(7 \mathrm{M})$ in the gel and formamide dissolving the sample serve as denaturants. Colored tracking dyes, such as bromphenol blue and xylene cyanol FF, can be loaded in the outside lanes to help determine the migration of the oligonucleotides in the inside lanes, which are not visible during electrophoresis. The relative migration rates of bromphenol blue and xylene cyanol FF to oligonucleotides changes as a function of acry-
Purification and Analysis of Synthetic Nucleic Acids and Components 
lamide concentration. Figure 10.4.1 shows the location of the dyes in a certain percentage of acrylamide relative to an oligonucleotide. The dyes determine the location of the oligonucleotides during electrophoresis and indicate the point at which the run should be terminated. For example, in a $15 \%$ gel, a $43-$-mer will run at the same rate as xylene cyanol FF.

UV shadowing, the visualization technique most commonly used for purification, can also be used for analysis of crude mixtures. UV shadowing is less labor-intensive than radiolabeling and often provides sufficient resolution for routine analysis. Although a relatively higher oligonucleotide concentration is required for visualization by UV-shadowing ( 0.5 to 2 OD units), this is only a fraction of a typical synthesis. Also, it is possible to utilize UV shadowing to analyze a preparative/purification gel run. A photograph can be taken under UV light for a permanent record. Oligonucleotides longer than $\sim 80 \mathrm{nt}$ often do not form well-shaped, detectable product bands, and should be analyzed by radiolabeling PAGE or gel capillary electrophoresis.

\section{Critical Parameters and Troubleshooting}

Sometimes the oligonucleotide sequence can have a marked affect on the resolution of the gel. Usually the problems are due to secondary structures of palindromic, self-complementary sequences and sequences with contiguous $\mathrm{G}$ nucleosides. Such oligonucleotides can show multiple or diffuse bands, which are nonreproducible. PAGE analysis of mixed-base oligonucleotides, where certain base positions contain a mix of 2 or more bases, also can be complex. The product is a collection of sequences of equal length, and may resolve into multiple bands or appear as a broad band. When purifying mixed-base oligonucleotides, a wide excision should be made to ensure recovery of all represented sequences.

Before the oligonucleotide undergoes electrophoresis, it should be quantitated by absorbance, desalted if necessary, and dried. The sample for PAGE is dissolved in $\sim 10 \mu \mathrm{L}$ per $1.5-\mathrm{cm}$ well for analytical-size gels $(0.8 \mathrm{~mm})$ of a mixture of formamide $/ 1 \times \mathrm{TBE}$ at a ratio of $9: 1$. This medium denatures, dissolves oligonucleotides rapidly, and is denser than $1 \times \mathrm{TBE}$, the electrophoresis buffer, so the sample falls through the buffer and settles on the bottom of the well and remains there until the power is turned on. Other denaturing loading media serve the same purpose. If an oligonucleotide is thought to form secondary structures, heating to $90^{\circ} \mathrm{C}$ for several minutes prior to loading may help to give normal elution patterns and a single product band.

Nucleic acid staining with dyes such as methylene blue or SYBR Green obviates the hazards and rapid decay of radioisotope labeling. Although ethidium bromide staining of DNA fragments is a well established technique for visualizing double-stranded DNA fragments, it is not useful for visualizing short, singlestranded DNA fragments such as oligonucleotides. The UV irradiation required to visualize ethidium bromide damages DNA and compromises the usefulness of this stain. Besides its toxicity hazards, the intercalation of ethidium bromide into short, single-stranded DNA is highly sequence-dependent and is sometimes undetectable in sequences less than $25 \mathrm{nt}$ long.

T4 polynucleotide kinase catalyzes the phosphorylation of an oligonucleotide $5^{\prime}$ hydroxyl by ATP (Wallace and Miyada, 1987). Detection of radiolabeled oligonucleotides is far more sensitive than the UV shadowing or staining methods. Desalting of the sample oligonucleotide is not necessary for UV shadow analysis but may be necessary prior to $3^{\prime}$ - or 5 '-end enzymatic radiolabeling. After radiolabeling, desalting or purification is not necessary prior to electrophoresis. The excess [ $\left.{ }^{32} \mathrm{P}\right] \mathrm{ATP}$ and other non-oligonucleotidic radioactive species will expose the film, but in most cases will not obscure the region of the labeled oligonucleotides of interest. If necessary, the radiolabeled oligonucleotides can be separated from the other components of the kinase reaction by gel-filtration, size-exclusion chromatography on Sephadex media (UNIT 10.7). The alternative radioisotope $\left[{ }^{33} \mathrm{P}\right]$ ATP is more stable (25 day half-life) and less hazardous (less penetrating); it is also available as $\left[\gamma^{-33} \mathrm{P}\right] \mathrm{ATP}$ and other nucleotides for labeling (NEN Life Science Products). Oligonucleotides with the DMTr group still on the $5^{\prime}$ end will not phosphorylate with kinase. Also, oligonucleotides with residual base-protecting groups remaining will appear as diffuse, slower-migrating species compared to deprotected samples, because the baseprotecting groups retard migration and add mass. Additionally, the base-protecting groups also hamper the ability of T4 kinase to end-label the product molecules. Excessive salt in the oligonucleotide sample may affect migration and may interfere with enzymatic labeling reactions. The presence of certain organic contaminants, such as acetic acid, can grossly distort electrophoretic mobility. Fresh kinase re- 
action buffer should be used, because this buffer contains DTT, which can degrade on storage.

For analyzing crude compounds, there must be an excess of ATP in the reaction for competitive labeling of each oligonucleotide species in a sample. With a limiting amount of ATP (excess of oligonucleotide), the kinase enzyme will preferentially phosphorylate oligonucleotides of $\sim 10$ to $20 \mathrm{nt}$ before it will phosphorylate longer sequences. This effect will skew the analysis and result in false representation of the sample composition. The usual amount of $\left[\gamma^{-32} \mathrm{P}\right]$ ATP is at a low concentration and is not sufficient by itself for efficient phosphorylation. The molar excess of ATP is provided by the addition of unlabeled (cold) ATP. Also, oligonucleotides can be $3^{\prime}$-radiolabeled by chain extension with radiolabeled nucleotides and terminal transferase (Sambrook et al., 1989). Other sample-related problems are due to inaccurate quantification of the sample, inactive enzyme, and inaccurate ATP concentrations. Oligonucleotide analogs, such as phosphorothioates, are not good substrates for kinase and will not radiolabel efficiently by phosphate transfer.

Autoradiography is used to visualize the electrophoresis of radiolabeled oligonucleotides. The radioactive oligonucleotide molecules emit $\beta$ particles which expose an $X$-ray film placed on top of the gel in the dark. To speed up the exposure process, an intensifying screen may be used. The film is developed in the dark with photographic developer and fixer and dried. Film exposure time is critical. An extended exposure will overrepresent failure sequences, since the product band will be saturated and will no longer respond to $\beta$ emissions. An exposure that is too short will not sufficiently reveal failure sequences. Figure 10.4.2 is an autoradiogram of a collection of crude ${ }^{32} \mathrm{P}$-labeled oligonucleotides, 70 to 140 nt. The amount of full-length oligonucleotide relative to shorter, failure sequences can be seen to decrease with increase in length. Careful quantitation of samples is necessary to make comparisons, since the dynamic range of the film is low. Once a band fully exposes the film, it does not further darken while the lighter bands containing less radioisotope continue to expose and darken the film. A useful alternative to autoradiography is phosphor imaging. In this technique, a reusable phosphor storage screen is exposed to the gel instead of an X-ray film. The storage screen is then laser-scanned and the emitted light is detected, digitized, and quantitated. Phosphor imaging is faster, more

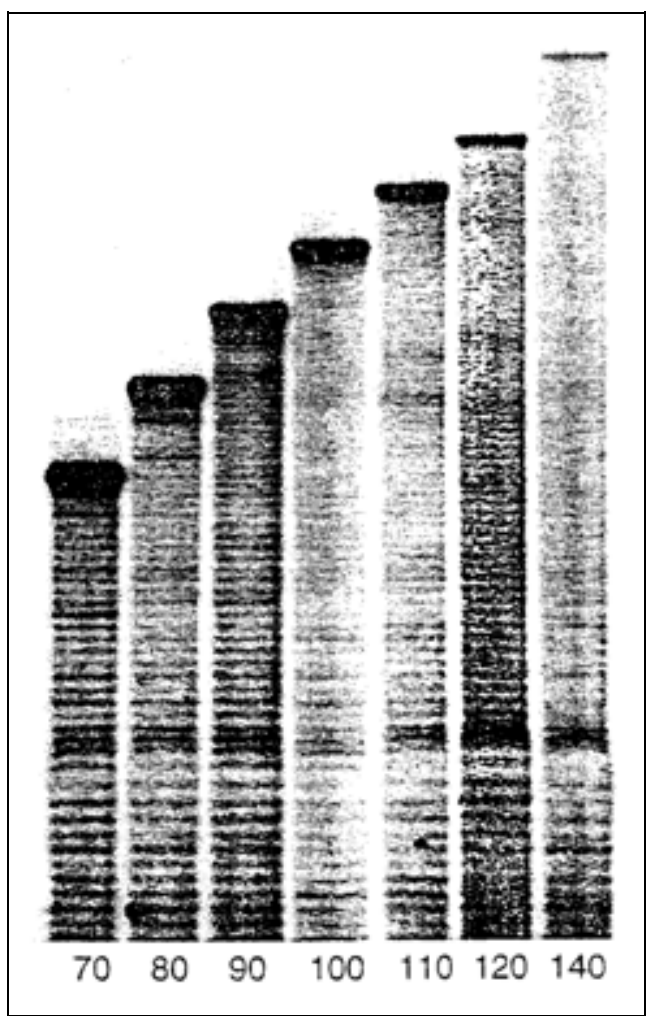

Figure 10.4.2 Autoradiogram of ${ }^{32} \mathrm{P}$-labeled ATP kinase gel, crude, 70- to 140-nt oligonucleotides.

sensitive, and provides a much broader dynamic range than autoradiography.

\section{Anticipated Results}

Following the above protocols, it can be expected that for average-length (20 to $40 \mathrm{nt}$ ) and reasonably pure crude oligonucleotides, single-base separation will be observed by PAGE. For example, a 30-nt oligonucleotide will be completely resolved from the mixture of N-1 29-nt failure sequences and visible as distinct bands. Radiolabeling will give better analytical results and resolution, especially for longer oligonucleotides. While PAGE has less capacity than HPLC for purification purposes, up to $1 \mathrm{mg}$ (30 OD units) of highly pure oligonucleotide can be isolated from a single gel.

\section{Time Considerations}

The protocols here can be completed in 1 to 2 days. Typical electrophoresis runs require 1 to $6 \mathrm{hr}$. The post-electrophoresis purification protocol usually requires an overnight treatment of soaking the gel pieces followed by 1 to $2 \mathrm{hr}$ for product isolation.
Purification and Analysis of Synthetic Nucleic Acids and Components 


\section{Literature Cited}

Andrus, A. 1992. Evaluating and Isolating Synthetic Oligodeoxynucleotides. PE Biosystems Division of Perkin-Elmer, Foster City, Calif. Available upon request.

Efcavitch, J.W. 1990. The electrophoresis of synthetic oligonucleotides. In Gel Electrophoresis of Nucleic Acids-A Practical Approach (D. Rickwood and B.D. Hames, eds.) pp. 125-149. Oxford University Press, Oxford.

Ellington, A and Pollard, J.D., Jr. 1998. Purification of oligonucleotides using denaturing polyacrylamide gel electrophoresis. In Current Protocols in Molecular Biology, vol. 1 (F. Ausubel, R. Brent, R.E. Kingston, D.D. Moore, J.G. Seidman, J.A. Smith, and K. Struhl, eds.) pp. 2.12.12.12.7. John Wiley \& Sons, New York.

Maniatis, T., Jeffrey, A., and van deSande, H. 1975. Chain length determination of small double- and single-stranded DNA molecules by polyacrylamide gel electrophoresis. Biochemistry 14:3787-3794.
Molecular Probes, 1996. Handbook of Fluorescent Probes and Research Chemicals, 6th ed., pp. 164.

Sambrook, J., Fritsch, E.F., and Maniatis, T. 1989. Molecular Cloning: A Laboratory Manual, 2nd ed. Cold Spring Harbor Laboratory, Cold Spring Harbor, N.Y.

Wallace, R.B. and Miyada, C.G. 1987. Oligonucleotide probes for the screening of recombinant DNA libraries. In Methods in Enzymology, Vol. 152, Guide to Molecular Cloning Techniques (S.L. Berger and A.R. Kimmel, eds.) pp. 432442. Academic Press, San Diego.

Contributed by Alex Andrus

PE Applied Biosystems

Foster City, California

Robert G. Kuimelis

Phylos, Inc.

Lexington, Massachusetts
Polyacrylamide Gel

(PAGE) of

Synthetic Nucleic Acids 